\title{
IMPROVING QUALITY OF SERVICE USING OFDM TECHNIQUE FOR 4TH GENERATION NETWORK
}

\author{
P.Sravan Kumar Reddy ${ }^{1}$, P.Suhail Parvaze ${ }^{2}$ \\ 1 Student (M.Tech), ECE Department, QUBA College of Engineering \& Technology, A.P., India, Sra1reddy@email.com \\ 2 Assoc.Prof, ECE Department, QUBA College of Engineering \& Technology, A.P., India, suhailsp@gmail.com
}

\begin{abstract}
The migration to $4 G$ networks will bring a new level of expectation to wireless communications. As after digital wireless revolution made mobile phones available for everyone, the higher speeds and packet delivery of $4 G$ networks will make high quality multimedia available everywhere. The key to achieving this higher level of service delivery is a new air interface, OFDM, which is in turn enabled by the high level of performance. OFDM provides a robust signal that requires relatively little power yet uses bandwidth very efficiently. Carriers will benefit from greater flexibility by using OFDM, since in the same spectrum they will be able to offer more channels, including higher 'bandwidth channels, with more types of services. Currently these systems are still being defined and prototyped. Achieving higher data rates requires OFDM systems to make more efficient use of the bandwidth than CDMA systems. One method of achieving this higher efficiency is through the use of higher order modulation. In this paper we have compared two digital modulation techniques 32QAM and 64QAM used for digital transmission of data. Our main objective to develop this configuration is to compare the performance of each modulation techniques. Comparison is done by Bit Error Rate analysis of both modulation techniques. We have developed existing configurations and improved them with high quality senders and receivers using MATLAB technology. In this paper we have also considered how OFDM can improve the real time video streaming over the wireless network. We have considered the problem of multiuser video streaming over OFDM. OFDM is a multi carrier modulation. The growing interest in Multicarrier Transmission by researchers and product developers motivated us to propose this topic for a special issue of Wireless Video transmission and Communications.
\end{abstract}

Index Terms: QAM' Quadrature Amplitude Modulation, OFDM' Orthogonal Frequency Division Multiplexing, QoS' Quality of service

\section{INTRODUCTION}

In OFDM, usable bandwidth is divided into a large number of smaller bandwidths that are mathematically orthogonal using fast Fourier transforms (FFTs). Reconstruction of the band is performed by the inverse fast Fourier transform (IFFT). One beneficial feature of this technique is the ease of adaptation to different bandwidths. The smaller bandwidth unit can remain fixed, even as the total bandwidth utilization is changed. For example, a $10^{\prime} \mathrm{MHz}$ bandwidth allocation may be divided into 1,024 smaller bands, whereas a $5^{\prime} \mathrm{MHz}$ allocation would be divided into 512 smaller bands. These smaller bands are referred to as subcarriers and are typically on the order of $10 \mathrm{kHz}$. One challenge in today's wireless systems is an effect called 'multipath.' Multipath results from reflections between a transmitter and receiver whereby the reflections arrive at the receiver at different times. The time span separating the reflection is referred to as delay spread. This type of interference tends to be problematic when the delay spread is on the order of the transmitted symbol time. Typical delay spreads are microseconds in length, which are close to CDMA symbol times. OFDMA symbol times tend to be on the order of 100 microseconds, making multipath less of a problem. In order to mitigate the effect of multipath, a guard band of about 10 microseconds, called the cyclic prefix, is inserted after each symbol. Achieving higher data rates requires OFDM systems to make more efficient use of the bandwidth than CDMA systems. The number of bits per unit hertz is referred to as the spectral efficiency. One method of achieving this higher efficiency is through the use of higher order modulation. Modulation refers to the number of bits that each subcarrier transmits. The design consideration of OFDM scheme has been discussed in past [1].

\section{RELATED WORK}

In the QAM digital modulation techniques, the Amplitude and Phase are the main parameters on which work is carried out. In our configuration we have taken AWGN channel (Gaussian Channel) where white Gaussian noise is added to the signal and that noisy signal is sent towards the receiver that is recovered with appropriate demodulator and decoders. The Wireless LAN standard, IEEE $802.11 \mathrm{~b}$, uses a variety of different QAMs depending on the data' rate required. QAM is employed, but has to be coupled with complementary code Modulation. The higher' speed wireless LANstandard, IEEE 802.11g has eight data rates: 6, 9, 12, 18, 24, 36, 48 and $54 \mathrm{Mbit} / \mathrm{s}$. The 6 and $9 \mathrm{Mbit} / \mathrm{s}$ modes use 
OFDM modulation where each sub'carrier is 8 QAM modulated. Many parameters for managing basic QoS for next generation network have been discussed in past [8] [10].

Multi'Carrier Modulation (MCM) is the principle of transmitting data by dividing the stream into several parallel bit streams, each of which has a much lower bit rate, and by using these sub streams to modulate several carriers. OFDM abandoned the use of steep band pass filters that completely separated the spectrum of individual subcarriers, as it was common practice in older Frequency Division Multiplex (FDMA) systems. OFDM time' domain waveforms are chosen such that mutual orthogonality is ensured even though subcarrier spectra may overlap. It appeared that such waveforms can be generated using a Fast Fourier Transform at the transmitter and receiver

\section{ORTHOGONAL FREQUENCY DIVESION}

\section{MULTIPLEXING (OFDM)}

OFDM is a combination technique between modulation and multiplexing. Modulation is a mapping of the information on changes in the carrier phase, frequency or amplitude or their combination. Meanwhile, multiplexing is a method of sharing a bandwidth with other independent data channel. In multiplexing, independent signals from different sources are sharing the channel spectrum. In OFDM, multiplexing is applied to independent signals but these independent signals are a sub'set of the one main signal. In OFDM the signal itself is first split into independent channels, modulated by data and then re'multiplexed to create the OFDM carrier. The multicarrier transmission technique uses the discrete Fourier transform. By using a DFT, the whole bandwidth will be split into $\mathrm{N}$ sub channels. As a result, a high data stream will be transformed into $\mathrm{N}$ low rate streams, which are transmitted over different sub'channels. OFDM symbols, which contain several modulation symbols, are formed as linear combinations of mutually orthogonal complex exponentials of finite duration [12]. The splitting of high rate data stream into a number of lower rate streams results in the increase of symbol duration. Mean while a lower rate parallel subcarriers reduces the relative amount of dispersion in time caused by multipath delay spread. Therefore OFDM is an advanced modulation technique which is suitable for high'speed data transmission due to its advantages in dealing with the multipath propagation problem and bandwidth efficiency. Fig: 1 show the spectrum of individual sub channel and the spectrum of the entire OFDM signal respectively. It can be noticed that there is no crosstalk from other channels at the center frequency of each subcarrier. As Fig: 2 shows the parallel transmission of data over multiple simultaneous carriers makes the OFDM system to be more robust against frequency selective fading or narrowband interference; some subcarriers may be degraded. a circle. With four phases, QAM can encode 32 bits per symbol, with Gray coding to minimize the BER - twice the rate of 8QAM. Analysis shows that this may be used either to double the data rate

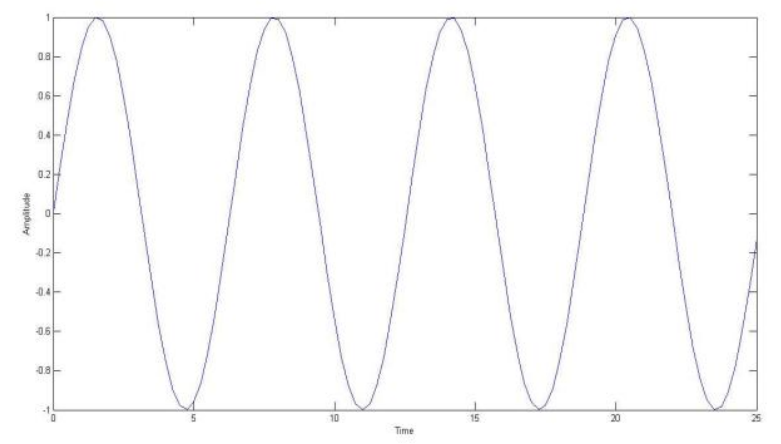

Fig 1: Frequency Plot for single carrier signal

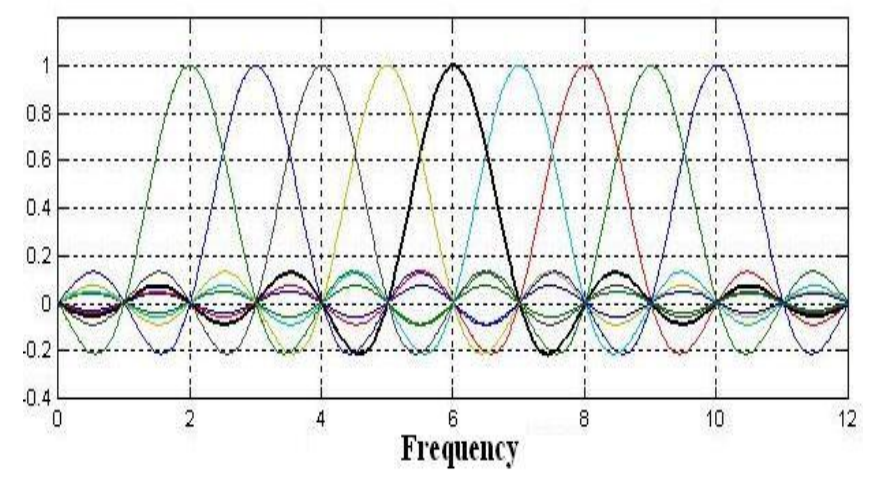

Fig 2 : Frequency Plot for Multi carrier signal

Like all modulation schemes, QAM conveys data by changing some aspect of a carrier signal, or the carrier wave, (usually a sinusoid) in response to a data signal. In the case of QAM, the amplitude of two waves, $90^{\circ}$ out-of-phase with each other (in quadrature) are changed (modulated or keyed) to represent the data signal. Amplitude modulating two carriers in quadrature can be equivalently viewed as both amplitude modulating and phase modulating a single carrier.

\subsection{QAM MODULATION}

A method for encoding digital data in an analog signal in which each combination of phase and amplitude represents one of sixteen four bit patterns. This is required for fax transmission at 9600 bits per second.Like all modulation schemes, QAM conveys data by changing some aspect of a carrier signal, or the carrier wave, (usually a sinusoid) in response to a data signal. In the case of QAM, the amplitude of two waves, $90^{\circ}$ out-ofphase with each other (in quadrature) are changed (modulated or keyed) to represent the data signal. 


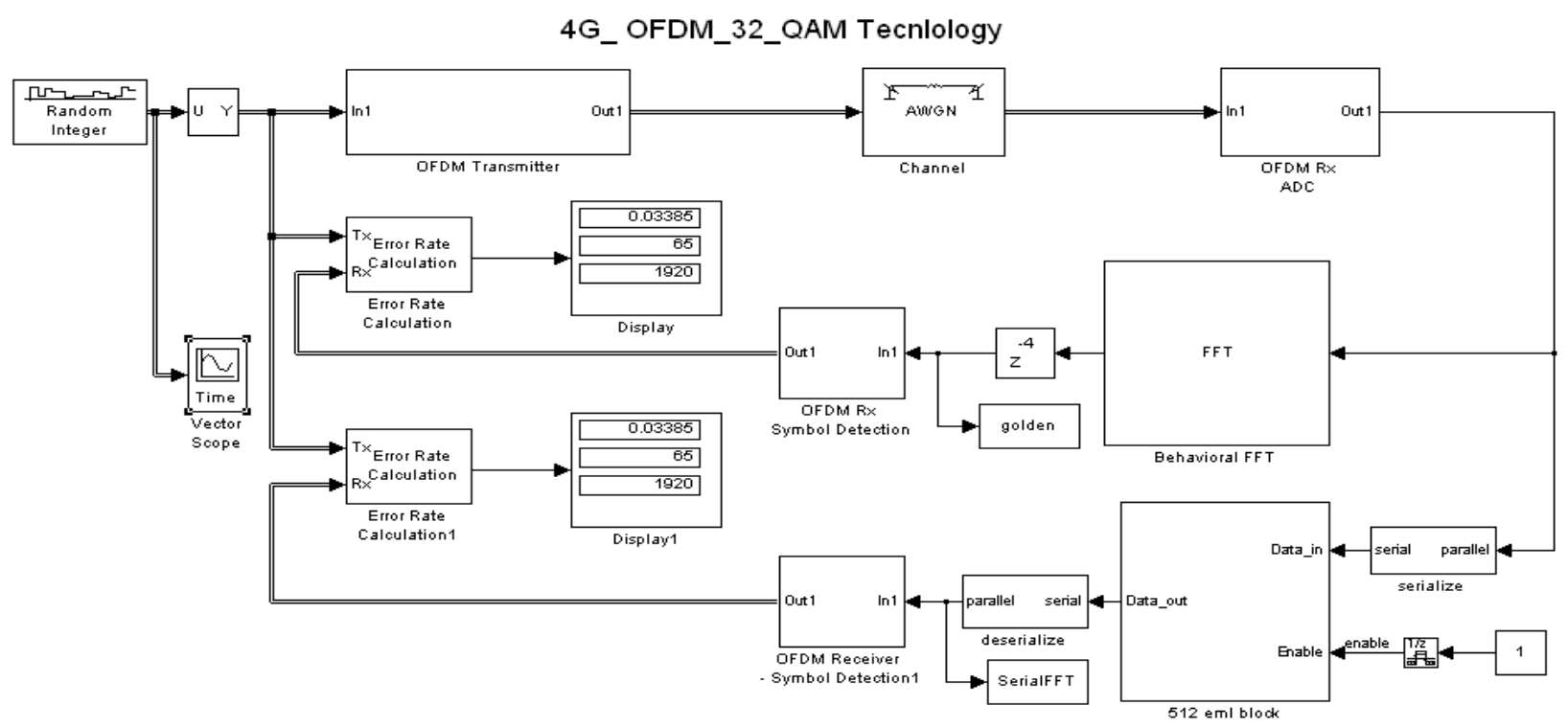

Fig 3: 4G_OFDM_32_QAM Technology

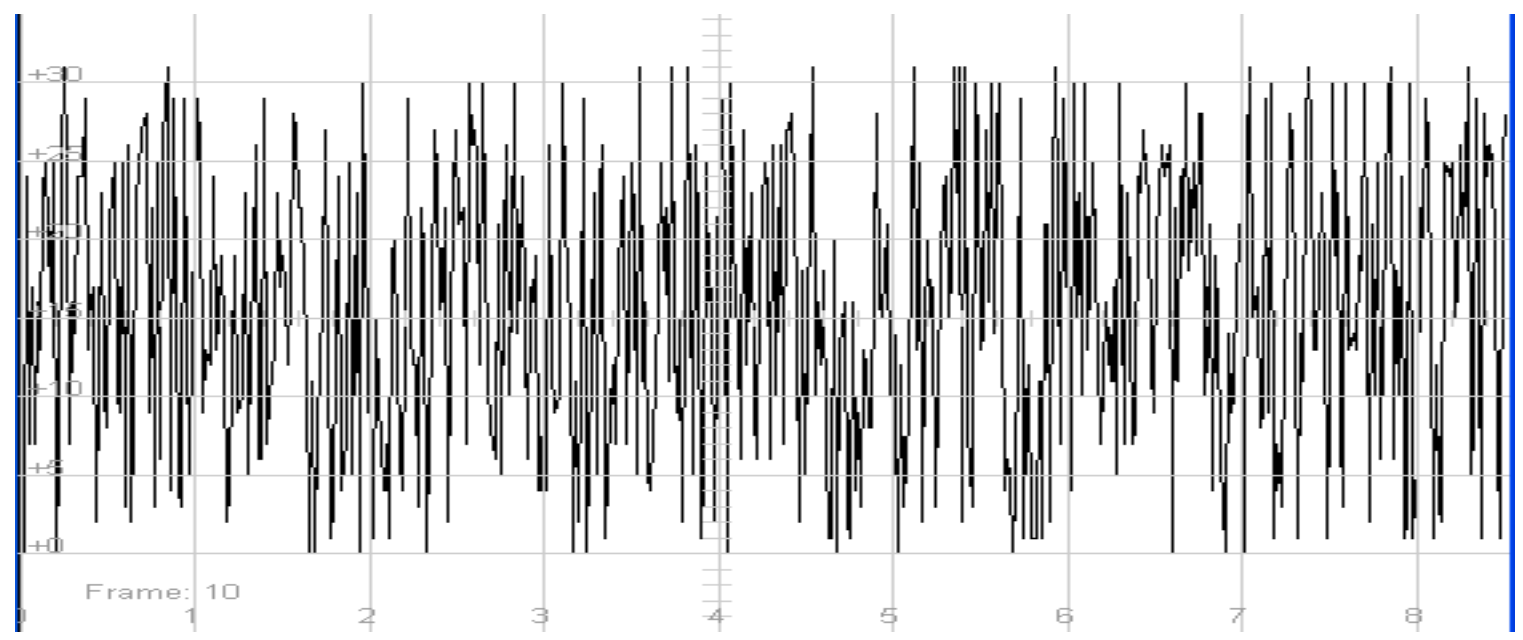

Fig 4: Input signal 10 Frames generated by source

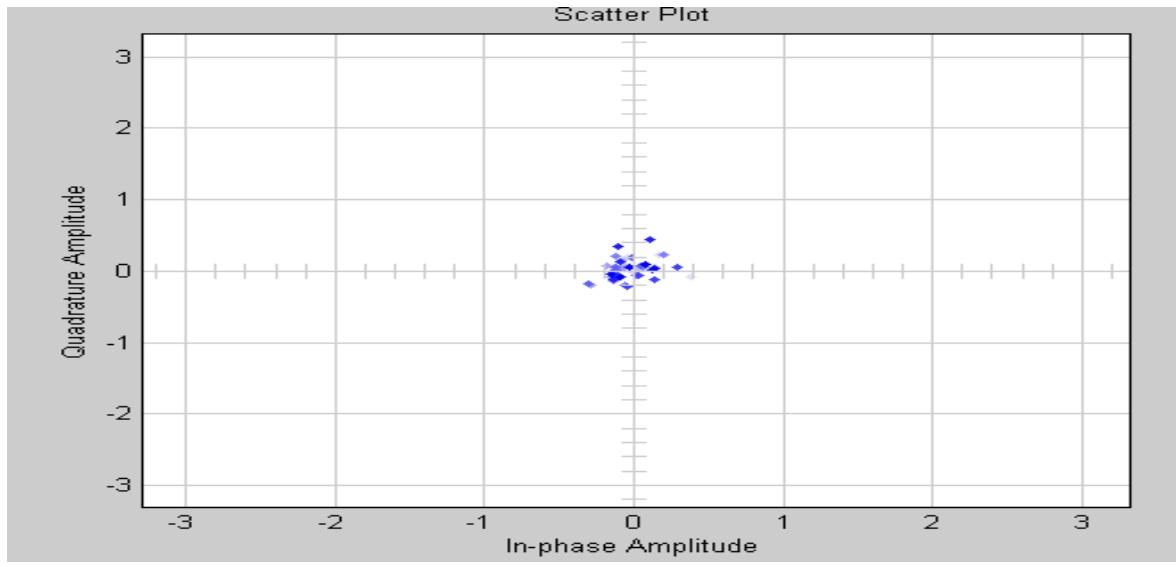

Fig 5: Scatter plot of OFDM_32_QAM For given source 


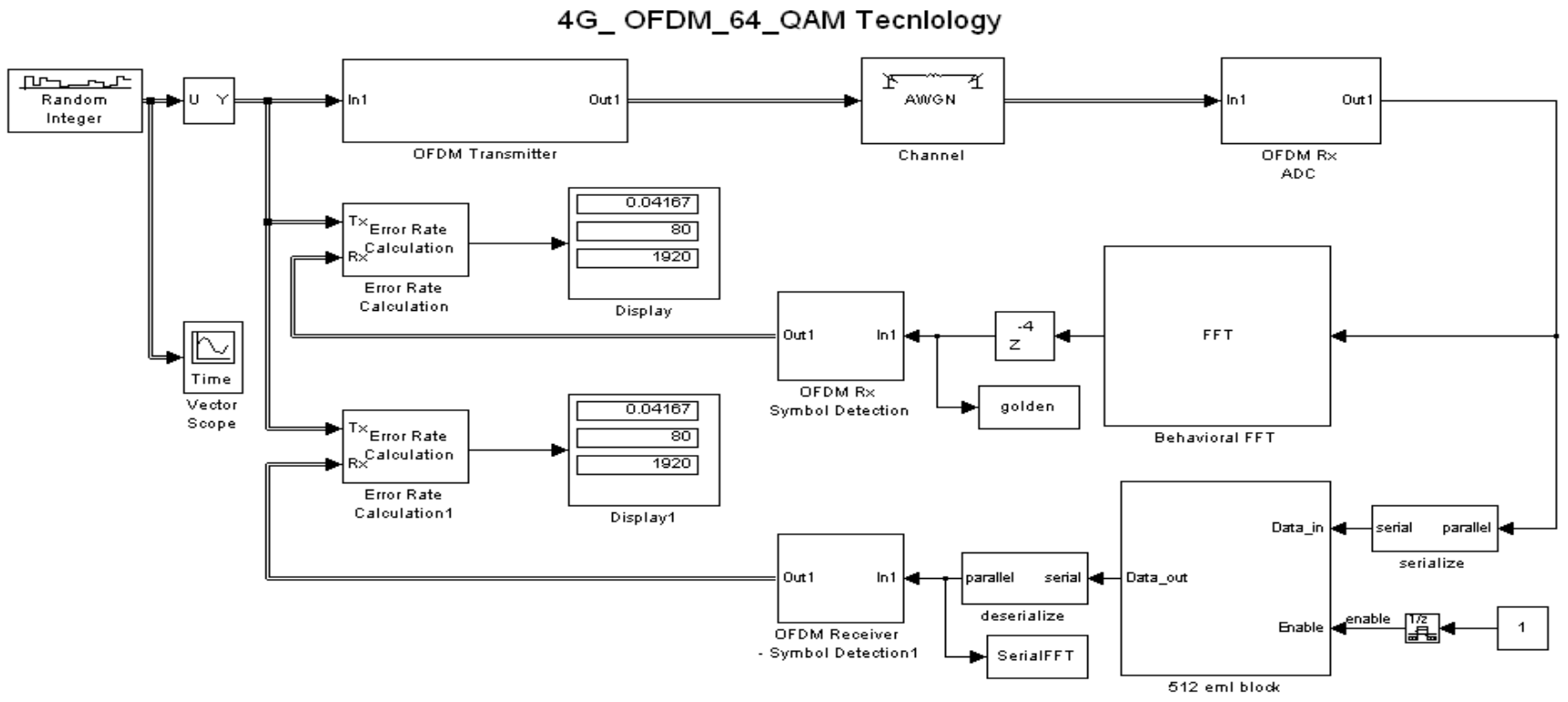

Fig 6: 4G_OFDM_64_QAM Technology

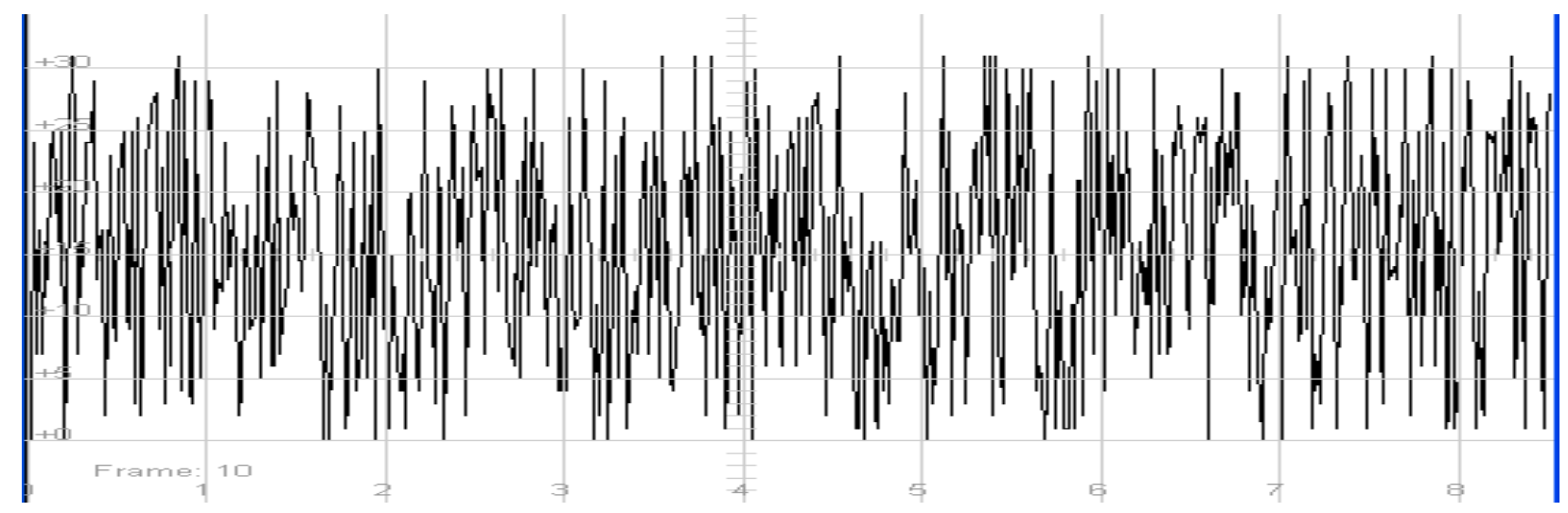

Fig 7: Input signal 10 Frames generated by source

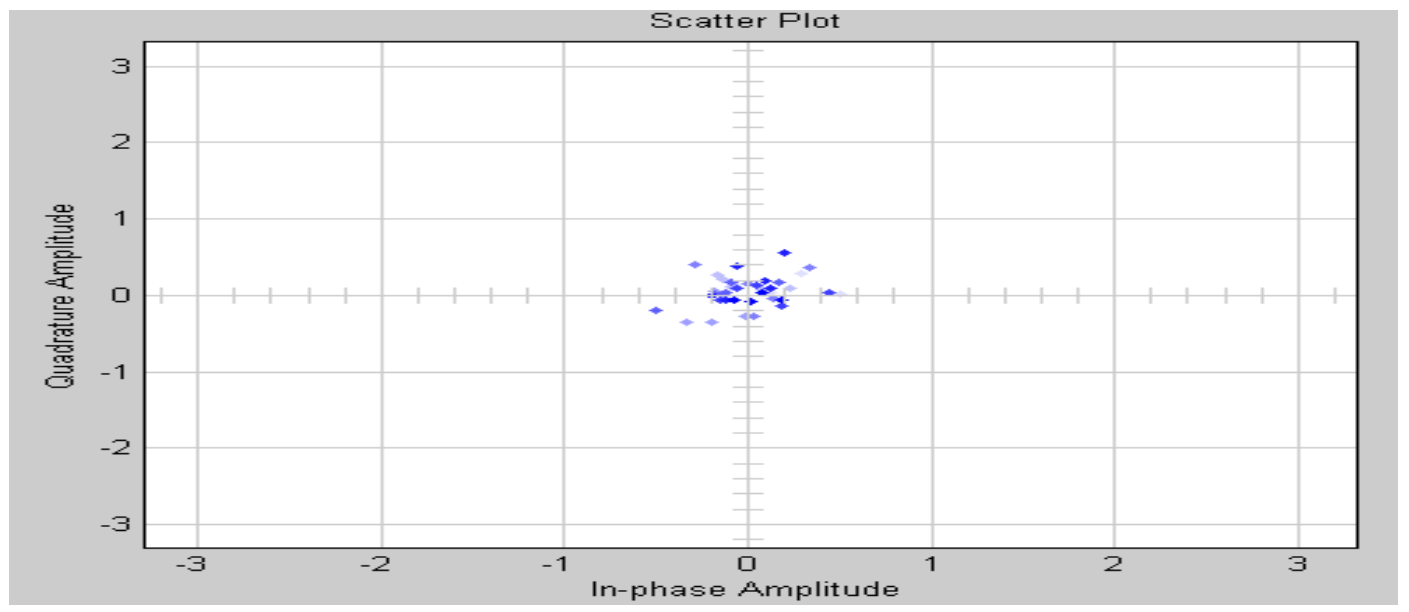


Amplitude modulating two carriers in quadrature can be equivalently viewed as both amplitude modulating and phase modulating a single carrier.

If data-rates beyond those offered by 8-PSK are required, it is more usual to move to QAM since it achieves a greater distance between adjacent points in the I-Q plane by distributing the points more evenly. The complicating factor is that the points are no longer all the same amplitude and so the demodulator must now correctly detect both phase and amplitude, rather than just phase. 64-QAM and 256-QAM are often used in digital cable television and cable modem applications. In the United States, 64-QAM and 256-QAM are the mandated modulation schemes for digital cable (see QAM tuner) as standardised by the SCTE in the standard ANSI/SCTE 07 2000. Note that many marketing people will refer to these as QAM-64 and QAM-256. In the UK, 16-QAM and 64-QAM are currently used for digital terrestrial television and 256-QAM is planned for Freeview-HD.

Communication systems designed to achieve very high levels of spectral efficiency usually employ very dense QAM constellations. For example current Homeplug AV2 500-Mbit power line Ethernet devices use 1024-QAM and 4096-QAM modulation, as well as future devices using ITU-T G.hn standard for networking over existing home wiring (coaxial cable, phone lines and power lines); 4096-QAM provides 12 bits/symbol. Another example is VDSL2 technology for copper twisted pairs, whose constellation size goes up to 32768 points.

\section{METHODOLOGY AND CHANNEL MODEL CONFIGURATION}

As mentioned in the introduction, in this paper we have compared the configuration of 32QAM and 64QAM techniques with OFDM technique. Methodology steps of the configuration are as follows:'

1.Data Source a. Select the random integer (in the range of 0 to 32 and 0 to 64 ).

b. Select the initial seed point (in the configuration we have taken 12345 as seed point)

c.Select the sample rate (192 samples/ frame in the configuration)

d.Convert the integer into bits (2bits per integer)

2. Modulation (32QAM/64QAM)

a. Apply the Modulation technique on generated

random data source. Output of the modulator

baseband is complex type.

b.Get the real part of the modulated data.

3.Apply OFDM modulation

a. Divide the carrier into subcarriers (10 sub carriers in the configuration)

b.Add cyclic redundancy for error detections and correction
c.Apply IFFT (Inverse Fast Fourier Transform) and take a conjugate symmetry to take real part of the transformed signal.

4.Add Noise to the carriers

a. Pass the signal over AWGN signal, this can be done by specifying Signal to Noise Ratio (SNR=15

in the configuration)

5. Apply OFDM demodulation

a. Remove the cyclic redundancy

b. Apply serial FFT (Fast Fourier Transform) to the signal as well as EML FFT algorithm

c. Convert the samples into frame

6. Demodulation (32QAM/64QAM)

a. Apply the demodulation technique on the output of

OFDM.

b. Convert the integer into bits

\section{PERFORMANCE EVALUATION}

In the presented model we have used Bit loss and packet loss evaluating the performance of both the modulation techniques with OFDM. We used MATLAB simulator to implement above model. Simulation shows that the signal is passed through noisy channel that is AWGN channel after OFDM modulation. Noise immunity can be altered in the signal and BER can be analyzed with different noise immunity. From the simulation results we observed that 64QAM modulation is more prone to errors while transmitting signal through noisy channel than 32QAM . Many performance evaluation of OFDM based system has been done earlier [2] [5].In our configuration 10 frames are transmitted, and each frame contains 192 bits, so total numbers of bits are transmitted as 1920.Table 1. shows comparative analysis between two modulation scheme using OFDM techniques. Result shows that 32 QAM scheme having minimum bit loss and packet loss so the 32QAM modulation scheme can be efficiently used for $4 \mathrm{G}$ networks

Table 1: Comparative Analysis

\begin{tabular}{|l|l|l|l|}
\hline Modulation & Total Bits & Bit Loss & Packet loss \\
\hline 32 QAM & 1920 & 65 & 0.03385 \\
\hline 64QAM & 1920 & 80 & 0.04167 \\
\hline
\end{tabular}

\section{REAL TIME VIDEO STREAMING WITH OFDM}

Video streaming has become a very popular application in communications as achievable data rates have improved dramatically. When real time video is transferred over the wireless network or real time video is displayed on the cellular phones then there are two issues that must be resolved 


\subsection{Bandwidth Requirement:}

Since the video transfer requires high data rate and high bandwidth Video requires large amount of data storage that is transferred over the network with limited bandwidth. This can be done by compressing the video before transmission.

\subsection{Modulation:}

Secondly very important issue is modulation of bits that are transferred. Because of the obstacles in the medium of the wireless data transmission the signal level can result in the loss of important information which must be perceived by the receiver. Streaming video is content sent in compressed form over the Internet and displayed by the viewer in real time. With streaming video or streaming media, a Web user does not have to wait to download a file to play it. Instead, the media is sent in a continuous stream of data and is played as it arrives. In our paper we have configured Orthogonal Frequency Division Multiplexing using MATLAB and SIMULINK. The main idea behind this is that divide the data to be sent into different blocks and each block is modulated with different sub carrier, and all the sub carries are orthogonal to each other. Results show a considerable amount of improvement in quality of video signals using OFDM techniques.

\section{CONCLUSIONS}

The demand for increased channel capacity in wireless and mobile communications has been rapidly increasing worldwide. The driving force behind the need to satisfy this requirement is the explosion in mobile telephone, Internet and multimedia services coupled with a limited radio spectrum. The BER is an important parameter in mobile communication for quality measurement of recovered data. In this paper we have presented a method to evaluate the performance of OFDM system with 32QAM and 64QAM. The comparison given in Table 1 and it shows the bit loss and packet loss of each modulation scheme. By comparison, it is observed that 32QAM modulation techniques having less packet loss and bit loss than 64QAM technique which enhance quality of experience to the user. Results have been obtained which is useful for channel parameter estimation and can be efficiently used for $4 \mathrm{G}$ networks.

\section{REFERENCES}

[1] Angela Dnufexi, Simon Armour, Andrew $N U$ and Mark Beach "Design considerations and initial physical layer cellular network" IEEE 2002 pp 192'196

[2] A. Dowler, A. Doufexi and A. Nix, "Performance Evaluation of Channel Estimation Techniques for a Mobile Fourth Generation Wide Area OFDM System," Vehicular Technology Conference, 2002.

Proceedings.VTC 2002 Fall, 2002 IEEE 56th, vol.4, pp. 2036 - 2040, 2002.

[3] Ahmed, A.B.; Osman, W.E.; Rahman, T.A.; Orthogonal "Frequency Division Multiple Acces System Analysis Using Bit Error Rate Next Generation Mobile Applications, Services and Technologies" 2009. NGMAST '09. Third International Conference on Publication Year: 2009 , Page(s): 211 ' 214

[4] Bit Error Rate: Fundamental Concepts and Measurement Issues by Gary Breed.

[5] Boudali Ouarzazi, Marion Berbineau, Iyad Dayoub, Atika Menhaj'Rivenq," Channel estimation of OFDM system for high data rate communications on mobile environments" IEEE Proceeding 2009 pp 425'429

[6] Gerhard Wunder, Thomas Michel, Chan Zhou, "Delay' Limited Transmission in OFDM Systems: Performance Bounds and Impact of System Parameters" IEEE Transactions on wireless communications, VOL. 8, NO. 7, JULY 2009 pp 3747'3757

[7] I. Korn, "Performance of Digital Communications Schemes with Multidimensional Signals in Gaussian Noise,” IEEE Trans. Commun., vol. 44, no. 9, September 1996.

[8] Kyung'chul Cho, Young'Chang Kang, Sun'hyung Kim "Performance Analysis of the DQPSK'OFDM System with Equalizer for the Wireless Multimedia Communication" Second International Conference on Future Generation Communication and Networking 2008 pp 78'81

[9] Neill Weilkinson "Next Generation Network Services Technologies and Strategies" john willey and sons ltd pp 167'170.

[10] Nor K. NoordinI, Borhanuddin M. AliI, S. S. Jamuarl and Mahamod B. Ismai12 Transmit Optimization for MIMO' OFDM With Delay'Constrained and No'Delay'Constrained Traffic IEEE transactions on signal processing, VOL. 54, NO. 8, AUGUST 2006

\section{BIOGRAPHIES:}

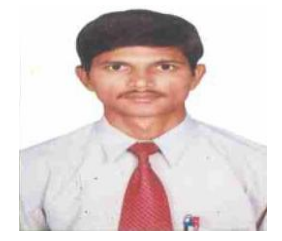

Anantapur, India

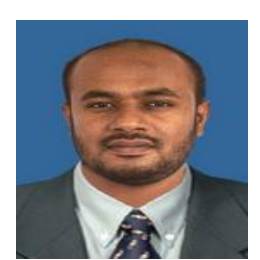

P. Sravan Kumar Reddy had Received B.Tech in Electronics and Communication Engineering (ECE) from JNTU, Hyderabad, India in 2007. He is persuing his M.Tech in Quba college of engineering and technology, JNTUA,

P.Suhail Parvaze is working $s$ an Associate Professor in QUBA College of engineering and Technology, Nellore. He has 7 years teaching experience both at UG and PG level and 1 year industrial experience 